\title{
ASYMPTOTICS OF VISIBILITY IN THE HYPERBOLIC PLANE
}

\author{
JOHAN TYKESSON,${ }^{*}$ Weizmann Institute of Science, Rehovot \\ PIERRE CALKA, ${ }^{* *}$ Université de Rouen
}

\begin{abstract}
At each point of a Poisson point process of intensity $\lambda$ in the hyperbolic plane, center a ball of bounded random radius. Consider the probability $P_{r}$ that, from a fixed point, there is some direction in which one can reach distance $r$ without hitting any ball. It is known (see Benjamini, Jonasson, Schramm and Tykesson (2009)) that if $\lambda$ is strictly smaller than a critical intensity $\lambda_{\mathrm{gv}}$ then $P_{r}$ does not go to 0 as $r \rightarrow \infty$. The main result in this note shows that in the case $\lambda=\lambda_{\mathrm{gv}}$, the probability of reaching a distance larger than $r$ decays essentially polynomially, while if $\lambda>\lambda_{\mathrm{gv}}$, the decay is exponential. We also extend these results to various related models and we finally obtain asymptotic results in several situations.
\end{abstract}

Keywords: Boolean model; hyperbolic geometry; visibility; Poincaré disc; Poisson line process

2010 Mathematics Subject Classification: Primary 60D05

Secondary 60G55

\section{Introduction}

We consider a general stationary random set in $\mathbb{R}^{d}$ which determines an occupied phase. An observer is located at the origin and tries to see as far as he/she can in each direction until his/her eyes meet the obstacle set. We say that percolation in visibility occurs when there is, with positive probability, an infinite geodesic ray included in the unoccupied phase, i.e. the observer can see to infinity in some direction. The total or maximal visibility is the length of the longest geodesic ray emanating from the origin and included in the outside of the random set. Its distribution will measure how close we stand from percolation in visibility. It can also be seen as a particular geometric characteristic which provides information on the random set itself and has been less studied than the classical chord and spherical contact distributions [1], [7], [14]. In this regard, the spherical contact distribution of the unoccupied phase could be renamed as the minimal visibility.

The main questions raised by such a model are: does percolation in visibility exist and is there a phase transition for it? What is the decrease of the distribution tail of the maximal visibility?

\footnotetext{
Received 7 January 2011; revision received 15 March 2012.

* Current address: Department of Mathematics, Uppsala University, Box 480, 75106 Uppsala, Sweden.

Email address: johan.tykesson@gmail.com

Research supported by a postdoctoral grant from the Swedish Research Council.

** Postal address: Laboratoire de Mathématiques Raphaël Salem, UMR 6085 CNRS-Université de Rouen, Avenue de l’Université, BP 12 Technopôle du Madrillet, F76801 Saint-Etienne-du-Rouvray, France.

Email address: pierre.calka@univ-rouen.fr
} 
The most natural and tractable random set is a homogeneous Poisson Boolean model in $\mathbb{R}^{d}$. It has been proved [4] that there is no percolation in visibility in any dimension and that the distribution tail of the total visibility decays exponentially fast while being logarithmically equivalent to the chord distribution, i.e. the visibility in a fixed direction. Roughly speaking, the space $\mathbb{R}^{d}$ is not large enoug $h$ far from the origin to secure percolation in visibility. Consequently, the easiest setting where a phase transition could occur is arguably the hyperbolic plane.

Hyperbolic spaces are classically connected with special relativity and astrophysics [21]. It has also recently been observed that large graphs in the Poincaré disc are well suited for applications to information visualization, in particular when systems with large hierarchies are considered [8], [15].

Let $X$ be a homogeneous Poisson point process with intensity $\lambda \in(0, \infty)$ in the hyperbolic plane $\mathbb{H}^{2}$. At each point of $X$, center a ball of a bounded random radius, independently for all points. When the radii are deterministic, equal to $R>0$, Kahane [12], [13] derived an explicit critical intensity $\lambda_{\mathrm{gv}}=(2 \sinh (R))^{-1}$ (the subscript 'gv' stands for 'geodesic vacant') with the following property. For $\lambda>\lambda_{\mathrm{gv}}$, the total visibility outside the union of the balls is finite almost surely, while, for $\lambda<\lambda_{\mathrm{gv}}$, with positive probability, there is some geodesic ray, starting at $o$, such that it does not hit any of the balls. In other words, if you stand at $o$ then, with positive probability, you have visibility to infinity inside the complement of the balls in some direction if the intensity is low enough. Of course, such a direction must be random, since in a given direction you will hit infinitely many balls with probability 1 . Lyons [16] generalized the result of Kahane to $d$-dimensional, complete, simply connected manifolds of negative curvature and in the case of constant negative curvature also found the exact value of the critical intensity. The existence of a critical intensity $\lambda_{\mathrm{gv}} \in(0, \infty)$ in the more general case of bounded random radii is contained in [2]. In particular, as soon as $\lambda \geq \lambda_{\mathrm{gv}}$, the set of visible points from $o$ are, with probability 1 , within some finite random distance.

In this note we mainly investigate the probability that there is some direction in which you can see to a distance larger than $r$ inside the complement of the balls, when $\lambda \geq \lambda_{\mathrm{gv}}$. In this region, the probability goes to 0 as $r$ approaches $\infty$, and here we are interested in what the decay looks like for large $r$. We will see that at the critical value this decay is essentially polynomial, while above criticality the decay is essentially exponential, with an exponent which differs from that of the decay for the visibility in a fixed direction. This also differs from the Euclidean case, where it is shown (see [4]) that, for every $\lambda>0$, one has exponential decay and the same decay as for the visibility in a fixed direction. We also generalize these results to visibility outside a Poisson process on the space of lines in $\mathbb{H}^{2}$. Indeed, Benjamini et al. [2] extended a previous work due to Porret-Blanc [18] to show that there is a critical intensity for the visibility to infinity in a Poisson line process in $\mathbb{H}^{2}$. In the same way as for the Boolean model, the decay of the distribution tail of the total visibility differs from the Euclidean case. Indeed, in [3], it has been shown that the distribution tail of the total visibility of the planar Euclidean Crofton cell is exponentially decreasing and logarithmically equivalent to the tail of the visibility in a fixed direction.

The rest of the paper is organized as follows. In Section 2 we introduce the model of main interest more carefully and state the main result, Theorem 2. In Section 3 we give the proof of Theorem 2. We then discuss some extensions of the main results to other models, in particular the line process model in Section 4. Section 5 provides the behavior of the total visibility near the critical point and for small intensity. In the last section, we show an asymptotic result when the size of the balls goes to 0 and the intensity increases accordingly. 


\section{Notation and main results}

Before turning to our results, we introduce the model more carefully. We will work in the Poincaré disc model of $\mathbb{H}^{2}$. This is the unit disc $\{z \in \mathbb{C}:|z|<1\}$ equipped with the metric

$$
\mathrm{d} s^{2}=4 \frac{\mathrm{d} x^{2}+\mathrm{d} y^{2}}{\left(1-\left(x^{2}+y^{2}\right)\right)^{2}} .
$$

Möbius transforms are isometries of $\mathbb{H}^{2}$; see (36) below. The associated area measure $\mu$ is isometry invariant:

$$
\mu(\mathrm{d} x, \mathrm{~d} y)=\frac{4}{\left(1-\left(x^{2}+y^{2}\right)\right)^{2}} \mathrm{~d} x \mathrm{~d} y .
$$

For more information about hyperbolic geometry, we refer the reader to [5]. Let us now describe the bounded radius version of the Poisson Boolean model of continuum percolation. We consider a homogeneous Poisson point process $X$ in $\mathbb{H}^{2}$, i.e. with intensity measure $\lambda \mu$, where $\lambda \in(0, \infty)$. Let $C \in(0, \infty)$, and suppose that $R$ is a random variable with $R \in(0, C]$ almost surely (a.s.). Let

$$
\mathcal{C}:=\bigcup_{x \in X} B\left(x, R_{x}\right)
$$

denote the occupied set, where $B(x, r)$ denotes the closed ball of radius $r$ centered at $x$ and $\left\{R_{x}\right\}_{x \in X}$ is a collection of independent and identically distributed (i.i.d.) random variables with the same distribution as $R$. Let

$$
\mathcal{W}:=\overline{\mathbb{H}^{2} \backslash \mathcal{C}} .
$$

We will call $\mathcal{W}$ the vacant set. It is well known that both $\mathcal{C}$ and $\mathcal{W}$ satisfy the property of positive correlations; see Theorem 2.2 of [17]. For $\mathcal{W}$, this means that, for any pair $f$ and $g$ of bounded increasing functions of $\mathcal{W}$, we have $\mathbb{E}[f(\mathcal{W}) g(\mathcal{W})] \geq \mathbb{E}[f(\mathcal{W})] \mathbb{E}[g(\mathcal{W})]$, and the definition for $\mathcal{C}$ is analogous.

This model has been extensively studied in Euclidean space; see, in particular, [6] and [17]. Aspects of the model have also recently been studied in hyperbolic space; see [2] and [20]. We will soon mention some of the results in [2], but first we introduce some notation.

For a set $A \subset \mathbb{H}^{2}$, let $A^{R}$ denote the closed $R$-neighborhood of $A$ :

$$
A^{R}=\{x: d(x, A) \leq R\} .
$$

By $c$ and $c^{\prime}$ we denote positive constants whose values may change from place to place, which may depend only on $\lambda$, the law of $R$, and the parameter $\epsilon$ introduced in Section 3. If they depend on some other parameter, this is indicated. By $\Theta(g)$ we denote a quantity which takes its values between $c g$ and $c^{\prime} g$. In addition, we define $\tilde{\Theta}(g)$ in the same way as $\Theta(g)$, but with the condition that $c$ and $c^{\prime}$ may not depend on $\lambda$.

Let $L_{r}(\theta)$ be the geodesic line segment started at 0 of length $r$ such that its continuation hits $\partial \mathbb{H}^{2}$ at the point $\mathrm{e}^{i \theta}$. For $\theta \in[0,2 \pi)$, the visibility in direction $\theta$ is defined as

$$
V(\theta)=\inf \left\{r \geq 0: L_{r}(\theta) \cap \mathcal{C} \neq \varnothing\right\} .
$$

The total visibility is defined to be

$$
\mathfrak{V}=\sup _{\theta \in[0,2 \pi)} V(\theta)
$$


Let $f(r)=f_{\lambda, R}(r)$ be the probability that a fixed line segment of length $r$ is contained in $\mathcal{W}$. Lemma 3.4 of [2] says that there is a unique $\alpha \geq 0$ such that

$$
f(r)=\Theta\left(\mathrm{e}^{-\alpha r}\right), \quad r \geq 0 .
$$

The constant $\alpha$ depends on the law of $R$ and on $\lambda$ and it can be computed explicitly; we will come back to this later. One of the main results in [2] is the following.

Theorem 1. For the total visibility $\mathfrak{V}$, the following statements hold:

$$
\mathbb{P}[\mathfrak{V}=\infty]=0 \quad \text { if } \alpha \geq 1, \quad \mathbb{P}[\mathfrak{V}=\infty]>0 \quad \text { if } \alpha<1 .
$$

We note that in [2], Theorem 1 was formulated in a much more general form. For example, visibility inside $\mathcal{C}$ was also dealt with.

In [2], the decay of $\mathbb{P}[\mathfrak{V} \geq r]$ as $r \rightarrow \infty$ in the case $\alpha \geq 1$ was not studied. One of the main results in this note provides upper and lower bounds as follows.

Theorem 2. For all large enough $r$,

$$
\mathbb{P}[\mathfrak{V} \geq r]=\Theta\left(\frac{1}{r}\right) \quad \text { if } \alpha=1, \quad \mathbb{P}[\mathfrak{V} \geq r]=\Theta\left(\mathrm{e}^{-(\alpha-1) r}\right) \quad \text { if } \alpha>1 .
$$

Finally, analogously to the determination of critical exponents in classical percolation, we can add some information about the behavior of the visibility set when the intensity is close to the critical value.

Theorem 3. When $\lambda \searrow \lambda_{\mathrm{gv}}$, we have

$$
\mathbb{E}[\operatorname{area}(\S)]=\frac{\tilde{\Theta}(1)}{\alpha-1} \text { and } \mathbb{E}[\mathfrak{V}]=\frac{\tilde{\Theta}(1)}{\alpha-1}
$$

\subsection{The value of $\alpha$}

If $R$ is nonrandom then Lemma 4.2 of [2] says that $\alpha=2 \lambda \sinh (R)$, i.e. $\lambda_{\mathrm{gv}}=(2 \sinh (R))^{-1}$. We can easily generalize this to the case when $R$ is random. For the convenience of the reader, we include the proof. For this particular result, we do not need $R$ to be bounded.

Lemma 1. If $R$ is random with $\mathbb{E}\left[\mathrm{e}^{R}\right]<\infty$ then the value of $\alpha$ is given by

$$
\alpha=2 \lambda \mathbb{E}[\sinh (R)] .
$$

In particular, $\lambda_{\mathrm{gv}}=1 / 2 \mathbb{E}[\sinh (R)]$.

Proof. Let $\gamma: \mathbb{R} \rightarrow \mathbb{H}^{2}$ be a line parameterized by the arc length, and let $r>0$. Let $\tilde{X} \subset X$ be the set of Poisson points $x \in X$ for which $B\left(x, R_{x}\right) \cap \gamma[0, r] \neq \varnothing$. If a Poisson point is at distance $t$ from $\gamma[0, r]$, the probability that its corresponding ball intersects $\gamma[0, r]$ is equal to $\mathbb{P}[R \geq t]$. Therefore, $\tilde{X}$ is a nonhomogeneous Poisson point process with intensity function $\Lambda(x)=\lambda \mathbb{P}[R \geq d(x, \gamma[0, r])]$. That is, for any measurable $A \subset \mathbb{H}^{2}$ and any nonnegative integer $k$,

$$
\mathbb{P}[|\tilde{X} \cap A|=k]=\exp \left(-\int_{A} \Lambda(x) \mathrm{d} \mu(x)\right) \frac{\left(\int_{A} \Lambda(x) \mathrm{d} \mu(x)\right)^{k}}{k !} .
$$


Observe that $\gamma[0, r] \subset \mathcal{W}$ if and only if $\tilde{X}=\varnothing$. Consequently, using Fubini's theorem,

$$
\begin{aligned}
f(r) & =\mathbb{P}[|\tilde{X}|=0] \\
& =\exp \left(-\int_{\mathbb{H}^{2}} \Lambda(x) \mathrm{d} \mu(x)\right) \\
& =\exp \left(-\lambda \int_{\mathbb{H}^{2}} \mathbb{P}[R \geq d(x, \gamma[0, r])] \mathrm{d} \mu(x)\right) \\
& =\exp \left(-\lambda \mathbb{E}\left[\mu\left(\gamma[0, r]^{R}\right)\right]\right) \\
& =\exp (-\lambda \mathbb{E}[2 \pi(\cosh (R)-1)+2 r \sinh (R)]),
\end{aligned}
$$

and the result follows. In the last equality we used the same calculation as used in the proof of Lemma 4.2 of [2].

\section{Proof of Theorem 2}

We now turn to the proof of Theorem 2. First we introduce some additional notation. For $\epsilon, \delta \in[0,2 \pi]$, let $Y_{r}(\epsilon, \delta)$ be the set $\left\{\theta \in[\epsilon, \delta]: L_{r}(\theta) \subset \mathcal{W}\right\}$. Note that, a.s., $Y_{r}(\epsilon, \delta)$ is a union of intervals. Let $y_{r}(\epsilon, \delta):=$ length $\left(Y_{r}(\epsilon, \delta)\right)$. Also, put $Y_{r}(\epsilon)=Y_{r}(0, \epsilon), y_{r}(\epsilon)=y_{r}(0, \epsilon)$, $Y_{r}:=Y_{r}(2 \pi)$, and $y_{r}:=y_{r}(2 \pi)$. Recall that $f(r)$ is the probability that a fixed line segment of length $r$ is contained in $\mathcal{W}$. Since the law of $\mathcal{W}$ is invariant under isometries of $\mathbb{H}^{2}$, we have $f(r)=\mathbb{P}\left[\theta \in Y_{r}\right]$ for every $\theta \in[0,2 \pi)$. For $x, y \in \mathbb{H}^{2}$, let $[x, y]$ be the line segment between $x$ and $y$, and, for $s>0$, let $[x, y]_{s}$ be the union of all line segments with one endpoint in $B(x, s)$ and the other endpoint in $B(y, s)$. Let $Q(x, y, s)$ be the event that $[x, y]_{s} \subset \mathcal{W}$.

Clearly, for each $s>0$,

$$
f(d(x, y)) \geq \mathbb{P}[Q(x, y, s)] .
$$

However, from Lemma 3.3 of [2], there exists some $c_{1}>0$ such that, for all small enough $s$ and all $x, y \in \mathbb{H}^{2}$,

$$
\mathbb{P}[Q(x, y, s)] \geq c_{1} f(d(x, y)) .
$$

If $R$ is fixed and we consider only intensities $\lambda$ within some compact interval, then $c_{1}$ can be chosen to be the same for all those values of $\lambda$, a fact we will make use of later. Lemma 3.3 of [2] states relation (3) for a larger class of random sets. We can remove the condition that $s$ is small enough, as we will see in the next lemma.

Lemma 2. For any $s \in(0, \infty)$, there is $c(s)>0$ such that, for all $x, y \in \mathbb{H}^{2}$,

$$
f(d(x, y)) \leq c(s) \mathbb{P}[Q(x, y, s)] .
$$

Proof. First we fix $s^{\prime}>0$ so small that (3) holds with $s^{\prime}$ in place of $s$. Let $s \in\left(s^{\prime}, \infty\right)$. Let $\gamma$ be a line parameterized by the arc length. Fix $r>0$ large, and let

$$
t_{1}:=\inf \left\{t: d\left(\gamma(t), \partial[\gamma(0), \gamma(r)]_{s}\right)<s^{\prime}\right\}
$$

From Lemma 3.2 of [2], we find that, for each $s>0$, there is some $c^{\prime}(s)<\infty$ which is independent of $r$ such that $t_{1}<c^{\prime}(s)$. In particular, $t_{1}$ does not diverge with $r$. Observe that, by the definition of $t_{1}$,

$$
Q(\gamma(0), \gamma(r), s) \supset Q\left(\gamma(0), \gamma\left(t_{1}\right), s\right) \cap Q\left(\gamma\left(t_{1}\right), \gamma\left(r-t_{1}\right), s^{\prime}\right) \cap Q\left(\gamma\left(r-t_{1}\right), \gamma(r), s\right) .
$$


By positive correlations and invariance, we obtain

$$
\mathbb{P}[Q(\gamma(0), \gamma(r), s)] \geq \mathbb{P}\left[Q\left(\gamma(0), \gamma\left(t_{1}\right), s\right)\right]^{2} \mathbb{P}\left[Q\left(\gamma\left(t_{1}\right), \gamma\left(r-t_{1}\right), s^{\prime}\right)\right] .
$$

We have

$$
\mathbb{P}\left[Q\left(\gamma\left(t_{1}\right), \gamma\left(r-t_{1}\right), s^{\prime}\right)\right] \geq \mathbb{P}\left[Q\left(\gamma(0), \gamma(r), s^{\prime}\right)\right] \stackrel{(3)}{\geq} c_{1} f(r)
$$

and

$$
\mathbb{P}\left[Q\left(\gamma(0), \gamma\left(t_{1}\right), s\right)\right]^{2} \geq \mathbb{P}\left[Q\left(\gamma(0), \gamma\left(c^{\prime}(s)\right), s\right)\right]^{2} .
$$

We now deduce (4) with $c(s)=c_{1} \mathbb{P}\left[Q\left(\gamma(0), \gamma\left(c^{\prime}(s)\right), s\right)\right]^{2}$ from (5), (6), and (7).

Equations (2) and (4) together imply that, for all $x, y \in \mathbb{H}^{2}$,

$$
\mathbb{P}[Q(x, y, s)]=\Theta(f(d(x, y))),
$$

where the implied constants in this case are allowed to depend on $s$. Theorem 2 is equivalent to the following estimate:

$$
\mathbb{P}\left[Y_{r} \neq \varnothing\right]= \begin{cases}\Theta\left(\mathrm{e}^{-(\alpha-1) r}\right) & \text { if } \alpha>1, \\ \Theta\left(r^{-1}\right) & \text { if } \alpha=1 .\end{cases}
$$

Recall that in [2] it was shown that in the case $\alpha<1$ there is positive probability that there are infinite rays contained in $\mathcal{W}$ emanating from 0 , so that $\mathbb{P}\left[Y_{r} \neq \varnothing\right]$ does not converge to 0 . We will make further remarks about the region $\alpha<1$ later. Observe that, by Fubini's theorem, we have

$$
\mathbb{E}\left[y_{r}(\epsilon)\right]=\epsilon \mathbb{P}\left[0 \in Y_{r}\right]=\epsilon f(r)
$$

and

$$
\mathbb{E}\left[y_{r}(\epsilon)^{2}\right]=\int_{0}^{\epsilon} \int_{0}^{\epsilon} \mathbb{P}\left[\theta \in Y_{r}, \theta^{\prime} \in Y_{r}\right] \mathrm{d} \theta \mathrm{d} \theta^{\prime} .
$$

Moreover, by invariance, it follows that

$$
\mathbb{P}\left[\theta \in Y_{r}, \theta^{\prime} \in Y_{r}\right]=\mathbb{P}\left[0 \in Y_{r},\left|\theta-\theta^{\prime}\right| \in Y_{r}\right] .
$$

Therefore, we have

$$
\begin{aligned}
\frac{\epsilon}{2} \int_{0}^{\epsilon / 2} \mathbb{P}\left[0 \in Y_{r}, \theta \in Y_{r}\right] \mathrm{d} \theta & \leq \int_{0}^{\epsilon} \int_{0}^{\epsilon} \mathbb{P}\left[\theta \in Y_{r}, \theta^{\prime} \in Y_{r}\right] \mathrm{d} \theta \mathrm{d} \theta^{\prime} \\
& \leq 2 \epsilon \int_{0}^{\epsilon} \mathbb{P}\left[0 \in Y_{r}, \theta \in Y_{r}\right] \mathrm{d} \theta .
\end{aligned}
$$

Denote by $J(r, \theta)$ the set $L_{r}(0) \cup L_{r}(\theta)$. Note that

$$
\mathbb{P}\left[0 \in Y_{r}, \theta \in Y_{r}\right]=\mathrm{e}^{-\lambda \mathbb{E}\left[\operatorname{area}\left(J(r, \theta)^{R}\right)\right]} .
$$

Since the area of $J(r, \theta)^{R}$ is increasing in $\theta$ on $[0, \pi]$, it follows that $\mathbb{P}\left[0 \in Y_{r}, \theta \in Y_{r}\right]$ is decreasing in $\theta$ on $[0, \pi]$ and, therefore, we have

$$
\int_{0}^{\epsilon / 2} \mathbb{P}\left[0 \in Y_{r}, \theta \in Y_{r}\right] \mathrm{d} \theta \geq \frac{1}{2} \int_{0}^{\epsilon} \mathbb{P}\left[0 \in Y_{r}, \theta \in Y_{r}\right] \mathrm{d} \theta .
$$

Observe that up to a set of measure 0 , the events $\left\{y_{r}>0\right\}$ and $\left\{Y_{r} \neq \varnothing\right\}$ are the same. The following lemma is the key ingredient for the proof of Theorem 2. 
Lemma 3. For every $r>0$ and every $\epsilon \in(0, \pi / 2)$,

$$
\frac{\mathbb{E}\left[y_{r}(\epsilon)\right]^{2}}{\mathbb{E}\left[y_{r}(\epsilon)^{2}\right]} \leq \mathbb{P}\left[Y_{r}(\epsilon) \neq \varnothing\right] \leq 4 \frac{\mathbb{E}\left[y_{r}(\epsilon)\right]^{2}}{\mathbb{E}\left[y_{r}(\epsilon)^{2}\right]} .
$$

For the proof of Lemma 3, we will use some techniques from [10] and [11].

Proof of Lemma 3. The lower bound is of course derived via the usual second moment method, so it remains to show the upper bound. The first part of the proof of the upper bound follows the method used in the proof of the lemma on page 146 of [11]. Fix some $\epsilon \in(0, \pi / 2)$. Let $A=A(r, \epsilon)$ be the event that $Y_{r}(\epsilon) \neq \varnothing$. First we show that

$$
\mathbb{E}\left[y_{r}(2 \epsilon)\right] \geq \mathbb{P}[A] \mathbb{E}\left[y_{r}(\epsilon) \mid 0 \in Y_{r}(\epsilon)\right],
$$

and then we deduce (13) from (14). Let $A_{N}=A_{N}(r, \epsilon)$ be the event that $Y_{r}(\epsilon)$ contains an interval of length at least $1 / N$. Then, clearly, $\mathbb{P}\left[A_{N}\right] \nearrow \mathbb{P}[A]$ as $N \nearrow \infty$. Fix an integer $N$. Let $A_{0}:=\left\{0 \in Y_{r}\right\}$, and, for $j=1, \ldots,[N \epsilon]$, let

$$
A_{j}:=\left\{0 \in Y_{r}^{\mathrm{c}}, \frac{1}{N} \in Y_{r}^{\mathrm{c}}, \ldots, \frac{j-1}{N} \in Y_{r}^{\mathrm{c}}, \frac{j}{N} \in Y_{r}\right\} .
$$

On $A_{N}$, exactly one of the events $A_{j}$ happens. We first argue that

$$
\mathbb{E}\left[y_{r}(2 \epsilon) \mathbf{1}_{A_{j}}\right] \geq \mathbb{E}\left[y_{r}\left(\frac{j}{N}, \frac{j}{N}+\epsilon\right) \mathbf{1}_{A_{j}}\right]=\mathbb{P}\left[A_{j}\right] \mathbb{E}\left[y_{r}(\epsilon) \mid 0 \in Y_{r}\right] .
$$

The left inequality is trivial. After division by $\mathbb{P}\left[A_{j}\right]$ we see that we need to show that

$$
\mathbb{E}\left[y_{r}\left(\frac{j}{N}, \frac{j}{N}+\epsilon\right) \mid A_{j}\right]=\mathbb{E}\left[y_{r}(\epsilon) \mid 0 \in Y_{r}\right] .
$$

By invariance, the right-hand side equals

$$
\mathbb{E}\left[y_{r}\left(\frac{j}{N}, \frac{j}{N}+\epsilon\right) \mid \frac{j}{N} \in Y_{r}\right] .
$$

Thus, it will suffice to show that, for each $\theta \in[j / N, j / N+\epsilon]$,

$$
\mathbb{P}\left[\theta \in Y_{r} \mid A_{j}\right]=\mathbb{P}\left[\theta \in Y_{r} \mid \frac{j}{N} \in Y_{r}\right] .
$$

So, fix some $\theta \in[j / N, j / N+\epsilon]$. We can write

$$
X=\bigcup_{i=1}^{4} X_{i}
$$

where

$$
\begin{gathered}
X_{1}=\left\{x \in X: B\left(x, R_{x}\right) \cap L_{r}\left(\frac{j}{N}\right) \neq \varnothing\right\} \\
X_{2}=\left\{x \in X: B\left(x, R_{x}\right) \cap L_{r}\left(\frac{j}{N}\right)=\varnothing, B\left(x, R_{x}\right) \cap L_{r}(\theta) \neq \varnothing\right\},
\end{gathered}
$$




$$
\begin{gathered}
X_{3}=\left\{x \in X: B\left(x, R_{x}\right) \cap L_{r}\left(\frac{j}{N}\right)=\varnothing, B\left(x, R_{x}\right) \cap\left(\bigcup_{i=0}^{j-1} L_{r}\left(\frac{i}{N}\right)\right) \neq \varnothing\right\}, \\
X_{4}=X \backslash \bigcup_{i=1}^{3} X_{i} .
\end{gathered}
$$

Note that $\left\{j / N \in Y_{r}\right\}=\left\{X_{1}=\varnothing\right\}$. Therefore, given that the event $\left\{j / N \in Y_{r}\right\}$ happens, the event $\left\{\theta \in Y_{r}\right\}$ is determined by $X_{2}$, and the event

$$
\tilde{A}:=\left\{0 \in Y_{r}^{\mathrm{c}}, \frac{1}{N} \in Y_{r}^{\mathrm{c}}, \ldots, \frac{j-1}{N} \in Y_{r}^{\mathrm{c}}\right\}
$$

is determined by $X_{3}$. Therefore, conditioned on $\left\{j / N \in Y_{r}\right\}$, the events $\tilde{A}$ and $\left\{\theta \in Y_{r}\right\}$ are conditionally independent, that is,

$$
\mathbb{P}\left[\tilde{A} \cap\left\{\theta \in Y_{r}\right\} \mid \frac{j}{N} \in Y_{r}\right]=\mathbb{P}\left[\tilde{A} \mid \frac{j}{N} \in Y_{r}\right] \mathbb{P}\left[\theta \in Y_{r} \mid \frac{j}{N} \in Y_{r}\right] .
$$

This implies that

$$
\mathbb{P}\left[\theta \in Y_{r} \mid \tilde{A} \cap\left\{\frac{j}{N} \in Y_{r}\right\}\right]=\mathbb{P}\left[\theta \in Y_{r} \mid \frac{j}{N} \in Y_{r}\right],
$$

which is the same as (16) and therefore (15) is established. After summing both sides of (15), we get

$$
\mathbb{E}\left[y_{r}(2 \epsilon)\right] \geq \mathbb{P}\left[A_{N}\right] \mathbb{E}\left[y_{r}(\epsilon) \mid 0 \in Y_{r}\right] .
$$

Letting $N \rightarrow \infty$ in (17) establishes (14). We next show, in a similar way as is done in [10], that

$$
\mathbb{E}\left[y_{r}(\epsilon) \mid Y_{r}(\epsilon) \neq \varnothing\right] \geq \frac{1}{2} \mathbb{E}\left[y_{r}(\epsilon) \mid 0 \in Y_{r}\right]
$$

This follows from

$$
\begin{aligned}
\mathbb{E}\left[y_{r}(\epsilon) \mid Y_{r}(\epsilon) \neq \varnothing\right] & =\frac{\mathbb{E}\left[y_{r}(\epsilon)\right]}{\mathbb{P}\left[Y_{r}(\epsilon) \neq \varnothing\right]} \\
& =\frac{\mathbb{E}\left[y_{r}(2 \epsilon)\right] / 2}{\mathbb{P}\left[Y_{r}(\epsilon) \neq \varnothing\right]} \\
& \stackrel{(14)}{\mathbb{P}\left[Y_{r}(\epsilon) \neq \varnothing\right] \mathbb{E}\left[y_{r}(\epsilon) \mid 0 \in Y_{r}\right] / 2} \\
& \mathbb{P}\left[Y_{r}(\epsilon) \neq \varnothing\right] \\
& =\frac{1}{2} \mathbb{E}\left[y_{r}(\epsilon) \mid 0 \in Y_{r}\right],
\end{aligned}
$$

where the second equality follows from invariance. We can now derive the upper bound in (13):

$$
\begin{array}{rll}
\mathbb{P}\left[Y_{r}(\epsilon) \neq \varnothing\right] & =\frac{\mathbb{E}\left[y_{r}(\epsilon)\right]}{\mathbb{E}\left[y_{r}(\epsilon) \mid Y_{r}(\epsilon) \neq \varnothing\right]} \\
& \stackrel{(18)}{\leq} & \frac{2 \mathbb{E}\left[y_{r}(\epsilon)\right]}{\mathbb{E}\left[y_{r}(\epsilon) \mid 0 \in Y_{r}\right]} \\
& =\frac{2 \mathbb{E}\left[y_{r}(\epsilon)\right]}{\int_{0}^{\epsilon} \mathbb{P}\left[\theta \in Y_{r} \mid 0 \in Y_{r}\right] \mathrm{d} \theta}
\end{array}
$$




$$
\begin{aligned}
& =\frac{2 \mathbb{E}\left[y_{r}(\epsilon)\right] \mathbb{P}\left[0 \in Y_{r}\right]}{\int_{0}^{\epsilon} \mathbb{P}\left[\theta \in Y_{r}, 0 \in Y_{r}\right] \mathrm{d} \theta} \\
& \stackrel{\text { (9), (11) }}{\leq} 4 \frac{\mathbb{E}\left[y_{r}(\epsilon)\right]^{2}}{\mathbb{E}\left[y_{r}(\epsilon)^{2}\right]} .
\end{aligned}
$$

This concludes the proof.

Proof of Theorem 2. In view of Lemma 3, we need to estimate $\mathbb{E}\left[y_{r}(\epsilon)^{2}\right]$. First we estimate $\mathbb{P}\left[0 \in Y_{r}, \theta \in Y_{r}\right]$ for $\theta \in(0, \epsilon]$ and $r>0$. We have

$$
\mathbb{P}\left[0 \in Y_{r}, \theta \in Y_{r}\right]=\mathbb{P}[J(r, \theta) \subset \mathcal{W}] .
$$

Let

$$
t_{\theta}:=\inf \left\{t: d\left(L_{\infty}(\theta) \backslash L_{t}(\theta), L_{\infty}(0)\right) \geq 2 C\right\} .
$$

That is, if a point $x \in L_{\infty}(\theta)$ is at a distance more than $t_{\theta}$ from the origin, the distance from $x$ to $L_{\infty}(0)$ is greater than or equal to $2 C$ (recall that if $d(A, B) \geq 2 C$ then $A \cap \mathcal{W}$ and $B \cap \mathcal{W}$ are independent). Below, we will consider events of the type $\left\{L_{r}(0) \backslash L_{s}(0) \subset \mathcal{W}\right\}$, and, if $s \geq r$, we will use the convention that such an event is the entire sample space.

We obtain

$$
\begin{aligned}
& \mathbb{P}[J(r, \theta) \subset \mathcal{W}] \\
& \quad=\mathbb{P}\left[\left\{J\left(r \wedge t_{\theta}, \theta\right) \subset \mathcal{W}\right\} \cap\left\{L_{r}(0) \backslash L_{t_{\theta}}(0) \subset \mathcal{W}\right\} \cap\left\{L_{r}(\theta) \backslash L_{t_{\theta}}(\theta) \subset \mathcal{W}\right\}\right] \\
& \quad \geq \mathbb{P}\left[J\left(r \wedge t_{\theta}, \theta\right) \subset \mathcal{W}\right] \mathbb{P}\left[L_{r}(0) \backslash L_{t_{\theta}}(0) \subset \mathcal{W}\right] \mathbb{P}\left[L_{r}(\theta) \backslash L_{t_{\theta}}(\theta) \subset \mathcal{W}\right],
\end{aligned}
$$

where the inequality follows from positive correlations. On the other hand,

$$
\begin{aligned}
\mathbb{P}[\{J & \left.\left.\left(r \wedge t_{\theta}, \theta\right) \subset \mathcal{W}\right\} \cap\left\{L_{r}(0) \backslash L_{t_{\theta}}(0) \subset \mathcal{W}\right\} \cap\left\{L_{r}(\theta) \backslash L_{t_{\theta}}(\theta) \subset \mathcal{W}\right\}\right] \\
& \leq \mathbb{P}\left[\left\{J\left(r \wedge t_{\theta}, \theta\right) \subset \mathcal{W}\right\} \cap\left\{L_{r}(0) \backslash L_{t_{\theta}+2 C}(0) \subset \mathcal{W}\right\} \cap\left\{L_{r}(\theta) \backslash L_{t_{\theta}+2 C}(\theta) \subset \mathcal{W}\right\}\right] \\
& =\mathbb{P}\left[J\left(r \wedge t_{\theta}, \theta\right) \subset \mathcal{W}\right] \mathbb{P}\left[L_{r}(0) \backslash L_{t_{\theta}+2 C}(0) \subset \mathcal{W}\right] \mathbb{P}\left[L_{r}(\theta) \backslash L_{t_{\theta}+2 C}(\theta) \subset \mathcal{W}\right] \\
& =\Theta(1) \mathbb{P}\left[J\left(r \wedge t_{\theta}, \theta\right) \subset \mathbb{W}\right] \mathbb{P}\left[L_{r}(0) \backslash L_{t_{\theta}}(0) \subset \mathcal{W}\right] \mathbb{P}\left[L_{r}(\theta) \backslash L_{t_{\theta}}(\theta) \subset \mathcal{W}\right],
\end{aligned}
$$

where we used independence at distance $2 C$ in the first equality. We also have

$$
\mathbb{P}\left[J\left(r \wedge t_{\theta}, \theta\right) \subset \mathcal{W}\right] \leq \mathbb{P}\left[L_{t_{\theta} \wedge r}(\theta) \subset \mathcal{W}\right] .
$$

Let $x(l)$ be the point on $L_{\infty}(0)$ which is at distance $l$ from $o$. Then we have

$$
\mathbb{P}\left[J\left(r \wedge t_{\theta}, \theta\right) \subset \mathcal{W}\right] \geq \mathbb{P}\left[Q\left(o, x\left(r \wedge t_{\theta}\right), 2 C\right)\right]=\Theta(1) \mathbb{P}\left[L_{r \wedge t_{\theta}}(0) \subset \mathcal{W}\right],
$$

where the last equality is due to Lemma 2. From (19), (20), (21), and (22), we obtain

$$
\begin{aligned}
\mathbb{P}[J(r, \theta) \subset \mathcal{W}] & =\Theta(1) \mathbb{P}\left[L_{r \wedge t_{\theta}}(0) \subset \mathcal{W}\right] \mathbb{P}\left[L_{r}(0) \backslash L_{t_{\theta}}(0) \subset \mathcal{W}\right] \mathbb{P}\left[L_{r}(\theta) \backslash L_{t_{\theta}}(\theta) \subset \mathcal{W}\right] \\
& =\Theta(1) f\left(r \wedge t_{\theta}\right) f\left(0 \vee\left(r-t_{\theta}\right)\right)^{2} .
\end{aligned}
$$

Let us prove that

$$
\mathbb{P}[J(r, \theta) \subset \mathcal{W}]=\Theta(1) f(r) f\left(0 \vee\left(r-t_{\theta}\right)\right) .
$$

From (23), it is enough to prove that

$$
f\left(r \wedge t_{\theta}\right) f\left(0 \vee\left(r-t_{\theta}\right)\right)^{2}=\Theta(1) f(r) f\left(0 \vee\left(r-t_{\theta}\right)\right) .
$$


Indeed, if $r \leq t_{\theta}$, we have

$$
f\left(r \wedge t_{\theta}\right) f\left(0 \vee\left(r-t_{\theta}\right)\right)^{2}=f(r)=f(r) f\left(0 \vee\left(r-t_{\theta}\right)\right) .
$$

Let us turn now to the case $r>t_{\theta}$ : we have

$$
f\left(r \wedge t_{\theta}\right) f\left(0 \vee\left(r-t_{\theta}\right)\right)^{2}=f\left(t_{\theta}\right) f\left(r-t_{\theta}\right)^{2} .
$$

From (1), let $C_{1}$ and $C_{2}$ be two positive constants (not depending on $r$ ) such that, for every $t>0$,

$$
C_{1} \mathrm{e}^{-\alpha t} \leq f(t) \leq C_{2} \mathrm{e}^{-\alpha t}
$$

Then

$$
\begin{aligned}
f\left(t_{\theta}\right) f\left(r-t_{\theta}\right)^{2} & \leq C_{2}^{3} \mathrm{e}^{-\alpha t_{\theta}} \mathrm{e}^{-2 \alpha\left(r-t_{\theta}\right)} \\
& =C_{2}^{3} \mathrm{e}^{-\alpha r} \mathrm{e}^{-\alpha\left(r-t_{\theta}\right)} \\
& \leq \frac{C_{2}^{3}}{C_{1}^{2}} f(r) f\left(r-t_{\theta}\right) .
\end{aligned}
$$

The same goes for bounding from below $f\left(t_{\theta}\right) f\left(r-t_{\theta}\right)^{2}$, so (24) is proved.

We deduce from (24) that

$$
\int_{0}^{\epsilon} \mathbb{P}\left[0 \in Y_{r}, \theta \in Y_{r}\right] \mathrm{d} \theta=\Theta(1) f(r) \int_{0}^{\epsilon} f\left(0 \vee\left(r-t_{\theta}\right)\right) \mathrm{d} \theta .
$$

We next investigate the behavior of $t_{\theta}$. Let $\gamma(t)$ be the geodesic which starts at 0 and then follows $L_{\infty}(\theta)$, and suppose that $\gamma(t)$ is parameterized by the arc length. Given $\theta$ and $t$, we first want to find the distance between $\gamma(t)$ and $L_{\infty}(0)$. Denote this distance by $s=s(t)$. By the hyperbolic law of cosines we have

$$
\cosh (2 s)=\cosh ^{2}(t)-\sinh ^{2}(t) \cos (2 \theta) .
$$

Using the identity $\cosh ^{2}(t)-\sinh ^{2}(t)=1$, we see that $s=2 C$ if and only if

$$
t=t_{\theta}=\cosh ^{-1}\left(\sqrt{\frac{\cosh (4 C)-\cos (2 \theta)}{1-\cos (2 \theta)}}\right) .
$$

A calculation shows that $\left(r-t_{\theta}\right)>0$ if and only if

$$
\theta>h(C, r):=\frac{1}{2} \cos ^{-1}\left(\frac{\cosh ^{2}(r)-\cosh (4 C)}{\cosh ^{2}(r)-1}\right)=\frac{1}{2} \cos ^{-1}\left(1-\frac{\cosh (4 C)-1}{\cosh ^{2}(r)-1}\right) .
$$

Now note that

$$
h(r, C)=\Theta(1) \mathrm{e}^{-r}
$$

for all large enough $r$ (using $\cos ^{-1}(1-x)=\sqrt{2 x}+O\left(x^{3 / 2}\right)$ for small $\left.x\right)$. Let

$$
\hat{t}(\theta):=\sqrt{\frac{\cosh (4 C)-\cos (2 \theta)}{1-\cos (2 \theta)}} .
$$


Using

$$
\cosh ^{-1}(x)=\log \left(x+\sqrt{x^{2}-1}\right) \in[\log (x), \log (x)+\log (2)), \quad x \geq 1,
$$

we obtain, for all large $r$,

$$
\begin{aligned}
\int_{0}^{\epsilon} f\left(0 \vee\left(r-t_{\theta}\right)\right) \mathrm{d} \theta & =\Theta(1)\left(\int_{0}^{h(C, r)} \mathrm{d} \theta+\int_{h(C, r)}^{\epsilon} \mathrm{e}^{-\alpha\left(r-t_{\theta}\right)} \mathrm{d} \theta\right) \\
& =\Theta(1)\left(h(C, r)+\mathrm{e}^{-\alpha r} \int_{h(C, r)}^{\epsilon} \mathrm{e}^{\alpha t_{\theta}} \mathrm{d} \theta\right) \\
& =\Theta(1)\left(h(C, r)+\mathrm{e}^{-\alpha r} \int_{h(C, r)}^{\epsilon}\left(\hat{t}(\theta)+\sqrt{\hat{t}(\theta)^{2}-1}\right)^{\alpha} \mathrm{d} \theta\right) .
\end{aligned}
$$

When $\theta$ goes to 0 , we deduce from (28) that

$$
\begin{aligned}
\hat{t}(\theta)+\sqrt{\hat{t}(\theta)^{2}-1} & \sim 2 \hat{t}(\theta) \\
& \sim 2 \sqrt{\cosh (4 C)-1}(1-\cos (2 \theta))^{-1 / 2} \\
& =\Theta(1)(1-\cos (2 \theta))^{-1 / 2},
\end{aligned}
$$

where we write $\varphi(\theta) \sim \psi(\theta)$ if $\lim _{\theta \rightarrow 0} \varphi(\theta) / \psi(\theta)=1$. Moreover, since

$$
1-\cos (2 \theta)=2 \theta^{2}+O\left(\theta^{3}\right) \in\left[\frac{4}{\pi} \theta^{2}, 2 \theta^{2}\right], \quad \theta \in\left[0, \frac{\pi}{4}\right],
$$

equality (31) implies that

$$
\hat{t}(\theta)+\sqrt{\hat{t}(\theta)^{2}-1}=\Theta(1) \theta^{-1} .
$$

We insert (27) and (32) into (30) to deduce that

$$
\begin{aligned}
\int_{0}^{\epsilon} f\left(0 \vee\left(r-t_{\theta}\right)\right) \mathrm{d} \theta & =\Theta(1)\left(\mathrm{e}^{-r}+\mathrm{e}^{-\alpha r} \int_{\Theta(1) \mathrm{e}^{-r}}^{\epsilon} \theta^{-\alpha} \mathrm{d} \theta\right) \\
& = \begin{cases}\Theta(1) \mathrm{e}^{-r}, & \alpha>1, \\
\Theta(1) r \mathrm{e}^{-r}, & \alpha=1 .\end{cases}
\end{aligned}
$$

Combining (33), (25), (11), and (12), we see that, for large $r$,

$$
\mathbb{E}\left[y_{r}(\epsilon)^{2}\right]= \begin{cases}\Theta(1) \mathrm{e}^{-(1+\alpha) r}, & \alpha>1, \\ \Theta(1) r \mathrm{e}^{-2 r}, & \alpha=1 .\end{cases}
$$

Note that

$$
\sum_{k=1}^{8} \mathbb{P}\left[Y_{r}\left(\frac{1}{4}(k-1) \pi, \frac{1}{4} k \pi\right) \neq \varnothing\right] \geq \mathbb{P}\left[Y_{r} \neq \varnothing\right] \geq \mathbb{P}\left[Y_{r}(\epsilon) \neq \varnothing\right] .
$$

Since $\mathbb{E}\left[y_{r}(\epsilon)\right]^{2}=\epsilon^{2} \mathrm{e}^{-2 \alpha r}$, Lemma 3, (34), and (35) imply that, for large $r$,

$$
\mathbb{P}\left[Y_{r}(\epsilon) \neq \varnothing\right]= \begin{cases}\Theta(1) \mathrm{e}^{-(\alpha-1) r} & \text { if } \alpha>1 \\ \Theta(1) \frac{1}{r} & \text { if } \alpha=1\end{cases}
$$

The result follows. 


\section{Generalizations of Theorem 2}

The proof of Theorem 2 in Section 3 can be fully or partially adapted to other settings than visibility inside $\mathcal{W}$. Here are some important cases.

\subsection{Random convex sets}

Let $K$ be a closed random convex set which contains the origin, such that the diameter of $K$ is a.s. less than $C<\infty$. In addition, assume that the law of $K$ is invariant under all rotations of $\mathbb{H}^{2}$. For $x \in \mathbb{H}^{2}$, let $\phi_{x}: \mathbb{H}^{2} \rightarrow \mathbb{H}^{2}$ be the Möbius transform mapping $x$ to 0 :

$$
\phi_{x}(z)=\frac{z-x}{1-\bar{x} z} .
$$

For each $x \in X$, let $K_{x}$ be an independent copy of $K$, and let

$$
\mathcal{C}_{K}=\bigcup_{x \in X} \phi_{x}^{-1}\left(K_{x}\right) \quad \text { and } \quad \mathfrak{w}_{K}=\overline{\mathbb{H}^{2} \backslash \mathcal{C}}
$$

It is easy to see that the proofs above for balls of random radius are adaptable to this more general case. All results from [2] used in the above proofs are also valid in this case. Thus, the conclusions of Theorem 2 and Proposition 1 remain true when replacing balls with random convex sets. The value of $\alpha$ will of course depend on the law of $K$. In this case we obtain, as in the proof of Lemma 1 ,

$$
f(r)=\exp \left(-\lambda \mathbb{E}\left[\mu\left(\left\{x: \phi_{x}^{-1}(K) \cap \gamma[0, r] \neq \varnothing\right\}\right)\right]\right) .
$$

To find the explicit value of $\alpha$, one has to calculate the expectation appearing in the exponent in (37).

\subsection{Asymptotics of visibility in the covered set}

It is also of interest to consider visibility inside the covered set $\mathcal{C}$. Let $\mathfrak{V}^{\prime}$ be the supremum of the set of $r \geq 0$ such that there is a line segment of length $r$ starting at the origin which is fully contained in $\mathcal{C}$. Let $h(r)$ be the probability that a fixed line segment of length $r$ is contained in $\mathcal{C}$. In [2], it was shown that there is some $\alpha^{\prime}$ such that $h(r)=\Theta\left(\mathrm{e}^{-\alpha^{\prime} r}\right)$. The lower bound in Theorem 2 is derived via the ordinary second moment method. Moreover, the calculations in the proof of Theorem 2 might be adapted to visibility inside $\mathcal{C}$, except where reference to Lemma 3 is made. The derivation of the upper bound in Lemma 3 does not go through for the covered set. In particular, we currently do not know how to prove (16). Consequently, at the moment we only know lower bounds as follows. There exist $c>0$ (depending on the law of the obstacles) and $r_{0}<\infty$ such that

$$
\begin{gathered}
\mathbb{P}\left[\mathfrak{V}^{\prime} \geq r\right] \geq c r^{-1} \quad \text { if } \alpha^{\prime}=1, r \geq r_{0}, \\
\mathbb{P}\left[\mathfrak{V}^{\prime} \geq r\right] \geq c \mathrm{e}^{-\left(\alpha^{\prime}-1\right) r} \quad \text { if } \alpha^{\prime}>1, r \geq r_{0} .
\end{gathered}
$$

\subsection{Asymptotics of visibility outside a Poisson line process}

We consider a Poisson line process in the Poincaré disc model of $\mathbb{H}^{2}$ defined as follows: we let $\mathcal{P}$ be a Poisson point process in the open unit disk with intensity measure

$$
\mu_{\lambda}(\mathrm{d} \rho, \mathrm{d} \theta)=2 \lambda \frac{\left(1+\rho^{2}\right)}{\left(1-\rho^{2}\right)^{2}} \mathrm{~d} \rho \mathrm{d} \theta .
$$


For every $x \in \mathcal{P}$, let $G_{x}$ be the hyperbolic line which contains $x$ and is orthogonal to the Euclidean line segment $[0, x]$. Let

$$
\mathcal{L}=\bigcup_{x \in \mathcal{P}} G_{x} .
$$

In particular, the law of $\mathcal{L}$ is invariant under rotations around 0 , and this will be used below without further mention.

In the same spirit as for the Boolean model, we denote by $Y_{r}(\epsilon), \epsilon \in[0,2 \pi], r \geq 0$, the set of all directions $\theta \in[0, \epsilon)$ such that the line $L_{r}(\theta)$ does not intersect $\mathcal{L}$. We keep the same notation $y_{r}(\epsilon)$ and $Y_{r}:=Y_{r}(2 \pi)$. In other words, $Y_{r}$ is the set of directions in which we reach distance $r$ without meeting any line from the Poisson line process.

In this setting, a value $\lambda_{C}$ for the intensity $\lambda$ is said to be critical if, for $\lambda>\lambda_{C}$, the probability of having $\bigcap_{r>0} Y_{r}(2 \pi)$ not empty (i.e. seeing in some direction up to $\infty$ ) is 0 and, for $\lambda<\lambda_{C}$, this same probability is positive. In [2], the existence of an explicit critical intensity equal to $\lambda=\frac{1}{2}$ has been proved. (In [2], a different but equivalent, up to scaling of the intensity measure, way of describing the Poisson line process was used. Therefore, the critical value there is 1 , rather than $\frac{1}{2}$.) In [18], an upper bound for the distribution tail of the maximal visibility was derived. We intend here to get a new more precise estimate as in Theorem 2.

In particular, we can show an analogue of Lemma 3: for every $r>0$ and $\epsilon \in(0, \pi / 2)$,

$$
\frac{\mathbb{E}\left[y_{r}(\epsilon)\right]^{2}}{\mathbb{E}\left[y_{r}(\epsilon)^{2}\right]} \leq \mathbb{P}\left[Y_{r}(\epsilon) \neq \varnothing\right] \leq 4 \frac{\mathbb{E}\left[y_{r}(\epsilon)\right]^{2}}{\mathbb{E}\left[y_{r}(\epsilon)^{2}\right]} .
$$

The proof of (38) can be written along the same lines. The main point is the extension of equality (16) to the setting of the Poisson line process. To do so, let us define

$$
M_{r}(\theta)=\left\{x \in \mathbb{H}^{2}: G_{x} \cap L_{r}(\theta) \neq \varnothing\right\} .
$$

Then, conditionally on $\left\{j / N \in Y_{r}\right\}$, the events $\left\{0 \in Y_{r}^{\mathrm{c}}, 1 / N \in Y_{r}^{\mathrm{c}}, \ldots,(j-1) / N \in Y_{r}^{\mathrm{c}}\right\}$ and $\left\{\theta \in Y_{r}\right\}$ are independent. Indeed, the first is determined by the intersection of the point process $\mathcal{P}$ with $\bigcup_{i=0}^{j-1} M_{r}(i / N) \backslash M_{r}(j / N)$, whereas the second is determined by the intersection of $\mathcal{P}$ with a disjoint set, namely, $M_{r}(\theta) \backslash M_{r}(j / N)$. This is sufficient to prove (13) and deduce (38).

We now use (38) to show our main theorem.

Theorem 4. When $r \rightarrow \infty$, we have

$$
\mathbb{P}\left[Y_{r} \neq \varnothing\right]= \begin{cases}\Theta(1) \mathrm{e}^{-(2 \lambda-1) r} & \text { if } \lambda>\frac{1}{2}, \\ \Theta(1) \frac{1}{r} & \text { if } \lambda=\frac{1}{2} .\end{cases}
$$

Proof. As for Theorem 2, the proof relies on the use of (38) and the estimation of both the first and second moments of $y_{r}(\epsilon)$.

By Equation 17.61 of [19] we have

$$
\mathbb{P}\left[0 \in Y_{r}\right]=\mathrm{e}^{-2 \lambda r} .
$$

Moreover,

$$
\mathbb{P}\left[0 \in Y_{r}, \theta \in Y_{r}\right]=\exp \left(-\lambda \operatorname{per}\left(T_{r, \theta}\right)\right),
$$

where per denotes the perimeter and $T_{r, \theta}$ is the hyperbolic triangle with apices $0, a_{r}$, and $b_{r}$, $a_{r}$ being the point on $L_{r}(0)$ at distance $r$ from the origin and $b_{r}$ being the point on $L_{r}(\theta)$ at distance $r$ from the origin.

We have

$$
\operatorname{per}\left(T_{r, \theta}\right)=2 r+\cosh ^{-1}\left(\cosh ^{2}(r)(1-\cos (\theta))+\cos (\theta)\right) .
$$


In particular, since $\cosh ^{-1}(t)=\log \left(t+\sqrt{t^{2}-1}\right)$ for every $t \geq 1$, we have

$$
\log (t) \leq \cosh ^{-1}(t) \leq \log (t)+\log (2), \quad t \geq 1 .
$$

Consequently, we deduce that, when $r \rightarrow \infty$,

$$
\begin{aligned}
\int_{0}^{\epsilon} \mathbb{P}\left[0 \in Y_{r}, \theta \in Y_{r}\right] \mathrm{d} \theta & =\Theta(1) \mathrm{e}^{-2 \lambda r} \int_{0}^{\epsilon}\left(\cosh ^{2}(r)(1-\cos (\theta))+\cos (\theta)\right)^{-\lambda} \mathrm{d} \theta \\
& =\Theta(1) \mathrm{e}^{-2 \lambda r} \int_{0}^{\epsilon}\left(\mathrm{e}^{2 r}+2+\cos (\theta)\left(2-\mathrm{e}^{2 r}\right)\right)^{-\lambda} \mathrm{d} \theta .
\end{aligned}
$$

Moreover, for any $\theta \in(0, \pi / 2), 1-\theta^{2} / 2 \leq \cos (\theta) \leq 1-\theta^{2} / \pi$. Replacing $\cos (\theta)$ in (39), we note that, for $C$ equal to 2 or $\pi$, we have

$$
\begin{aligned}
\int_{0}^{\epsilon}\left(\mathrm{e}^{2 r}+2+\left(1-\frac{\theta^{2}}{C}\right)\left(2-\mathrm{e}^{2 r}\right)\right)^{-\lambda} \mathrm{d} \theta & =\int_{0}^{\epsilon}\left(4+\theta^{2}\left(\frac{\mathrm{e}^{2 r}}{C}-\frac{2}{C}\right)\right)^{-\lambda} \mathrm{d} \theta \\
& =\left(\frac{\mathrm{e}^{2 r}}{C}-\frac{2}{C}\right)^{-1 / 2} \int_{0}^{\epsilon \sqrt{\mathrm{e}^{2 r}-2} / \sqrt{C}} \frac{\mathrm{d} \theta}{\left(4+\theta^{2}\right)^{\lambda}} \\
& = \begin{cases}\Theta(1) \mathrm{e}^{-r} \int_{0}^{\infty} \frac{\mathrm{d} \theta}{\left(1+\theta^{2}\right)^{-\lambda}} & \text { if } \lambda>\frac{1}{2}, \\
\Theta(1) \mathrm{e}^{-r} r & \text { if } \lambda=\frac{1}{2} .\end{cases}
\end{aligned}
$$

Inserting this last result into (39) yields

$$
\int_{0}^{\epsilon} \mathbb{P}\left[0 \in Y_{r}, \theta \in Y_{r}\right] \mathrm{d} \theta= \begin{cases}\Theta(1) \mathrm{e}^{-(2 \lambda+1) r} & \text { if } \lambda>\frac{1}{2}, \\ \Theta(1) \mathrm{e}^{-2 r} r & \text { if } \lambda=\frac{1}{2} .\end{cases}
$$

We conclude by inserting (40) into (38).

\section{Critical point and small intensity}

\subsection{When $\alpha \rightarrow 1$}

In this section we study the behavior of the visibility near the critical point $\lambda=\lambda_{\mathrm{gv}}$. Recall that $\mathfrak{V}$ is the total visibility, i.e. $\mathfrak{V}=\sup \left\{r>0: Y_{r} \neq \varnothing\right\}$. Let

$$
s=\left\{x \in \mathbb{H}^{2}:[0, x] \subset \mathcal{W}\right\}
$$

be the set of all points visible from the origin. The set $\delta$ is sometimes called the visibility star. Recall that $\alpha=2 \lambda \mathbb{E}[\sinh (R)]$.

Proof of Theorem 3. Fix $\lambda_{1}>\lambda_{\mathrm{gv}}$. We now verify that $\Theta(1)$ in both (1) and in (8) stay in $(0, \infty)$ when $\lambda \in\left[0, \lambda_{1}\right]$, which implies that they can be replaced by $\tilde{\Theta}(1)$ when $\lambda \in\left[0, \lambda_{1}\right]$.

Indeed, we get from the proof of Lemma 3.4 of [2] that the constant in (1) is between $f(2 C)$ and 1 and the quantity $f(2 C) \in(0,1]$ for $\lambda \leq \lambda_{1}$. As for $\Theta(1)$ in (8), it is deduced from displays (20), (22), and (24).

- In (20), we have

$$
\begin{aligned}
\mathbb{P}\left[L_{r}(0) \backslash L_{t_{\theta}}(0) \subset \mathcal{W}\right] & =\mathbb{P}\left[\left\{L_{r}(0) \backslash L_{t_{\theta}+2 C}(0) \subset \mathcal{W}\right\} \cap\left\{L_{t_{\theta}+2 C}(0) \backslash L_{t_{\theta}}(0) \subset \mathcal{W}\right\}\right] \\
& \geq \mathbb{P}\left[L_{r}(0) \backslash L_{t_{\theta}+2 C}(0) \subset \mathcal{W}\right] \mathbb{P}\left[L_{t_{\theta}+2 C}(0) \backslash L_{t_{\theta}}(0) \subset \mathcal{W}\right] \\
& =\mathbb{P}\left[L_{r}(0) \backslash L_{t_{\theta}+2 C}(0) \subset \mathcal{W}\right] \mathbb{P}\left[L_{2 C}(0) \subset \mathcal{W}\right] .
\end{aligned}
$$

It suffices to see now that $\mathbb{P}\left[L_{2 C}(0) \subset \mathcal{W}\right]=f(2 C) \in(0,1]$ for $\lambda \leq \lambda_{1}$. 
- In (22), the constant comes from the calculation of $\mathbb{P}[Q(x, y, s)], x, y \in \mathbb{H}^{2}, s>0$. Let us consider

$$
\mathcal{E}\left([x, y]_{s}, R\right)=\left\{z \in \mathbb{H}^{2}: B(z, R) \cap[x, y]_{s} \neq \varnothing\right\} .
$$

Then

$$
\begin{aligned}
\mathbb{P}[Q(x, y, s)] & =\mathrm{e}^{-\lambda \operatorname{area}\left(\mathcal{E}\left([x, y]_{s}, R\right)\right)} \\
& =\mathrm{e}^{-\lambda\left(\operatorname{area}\left(\mathcal{E}\left([x, y]_{s}, R\right)\right)-\operatorname{area}\left(\mathcal{E}\left([x, y]_{0}, R\right)\right)\right)} f(d(x, y)) .
\end{aligned}
$$

It remains to note that $\mathrm{e}^{-\lambda\left(\operatorname{area}\left(\mathcal{E}\left([x, y]_{s}, R\right)\right)-\operatorname{area}\left(\mathcal{E}\left([x, y]_{0}, R\right)\right)\right)} \in(0,1]$ when $\lambda \leq \lambda_{1}$.

- In (24), the constant is between $f(4 C)$ and 1 because, when $r>t_{\theta}$, we have

$$
f(r) f(4 C) \leq f\left(t_{\theta}\right) f\left(r-t_{\theta}\right) \leq f(r) .
$$

Now, a classical use of Fubini's theorem and (1) yields

$$
\begin{aligned}
\mathbb{E}[\operatorname{area}(8)] & =2 \pi \int_{0}^{1} f\left(\log \left(\frac{1+r}{1-r}\right)\right) \frac{4 r \mathrm{~d} r}{\left(1-r^{2}\right)^{2}} \\
& =\tilde{\Theta}(1) \int_{0}^{1}\left(\frac{1+r}{1-r}\right)^{\alpha} \frac{r \mathrm{~d} r}{(1-r)^{2}(1+r)^{2}} \\
& =\tilde{\Theta}(1) \int_{0}^{1} \frac{\mathrm{d} r}{(1-r)^{2-\alpha}} \\
& =\frac{\tilde{\Theta}(1)}{\alpha-1} \quad \text { when } \lambda \searrow \lambda_{\mathrm{gv} .}
\end{aligned}
$$

In the same way, the second estimate is obtained with the use of (8):

$$
\mathbb{E}[\mathfrak{V}]=\int_{0}^{\infty} \mathbb{P}[\mathfrak{V} \geq r] \mathrm{d} r=\int_{0}^{\infty} \mathbb{P}\left[Y_{r} \neq \varnothing\right] \mathrm{d} r=\frac{\tilde{\Theta}(1)}{\alpha-1}, \quad \lambda \searrow \lambda_{\mathrm{gv}} .
$$

This completes the proof.

We conclude the section by studying how the probability to see to infinity increases as $\lambda$ decreases from $\lambda_{\mathrm{gv}}$. It was proved in Lemma 1 that $\alpha=\alpha(\lambda)=2 \lambda \mathbb{E}[\sinh (R)]$. In the following proposition, we will write $\alpha(\lambda)$ for $\alpha$ in order to make it clear that the asymptotic estimate is indeed a function of $\lambda$.

Proposition 1. For $\lambda \in\left[0, \lambda_{\mathrm{gv}}\right]$ and $\epsilon>0$,

$$
\mathbb{P}\left[Y_{\infty}(\epsilon) \neq \varnothing\right]=\tilde{\Theta}(1)(1-\alpha(\lambda)) .
$$

Proof. Repeating the calculations leading to (33) and (34), and using the fact that from the proof of Proposition 3 we can replace $\Theta(1)$ with $\Theta(1)$ at appropriate places, it follows that

$$
\int_{0}^{\epsilon} f\left(0 \vee\left(r-t_{\theta}\right)\right) \mathrm{d} \theta=\tilde{\Theta}(1)\left(\mathrm{e}^{-r}+\frac{\mathrm{e}^{-\alpha r}}{1-\alpha}-\frac{\mathrm{e}^{-r}}{1-\alpha}\right)
$$

and

$$
\mathbb{E}\left[y_{r}(\epsilon)^{2}\right]=\tilde{\Theta}(1)\left(\mathrm{e}^{-(1+\alpha) r}+\frac{\mathrm{e}^{-2 \alpha r}}{1-\alpha}-\frac{\mathrm{e}^{-(1+\alpha) r}}{1-\alpha}\right)=\tilde{\Theta}(1) \frac{\mathrm{e}^{-2 \alpha r}}{1-\alpha},
$$

where the last equality follows since $\alpha<1$. Using $\mathbb{E}\left[y_{r}(\epsilon)\right]^{2}=\epsilon^{2} \mathrm{e}^{-2 \alpha r}$, Lemma 3, and letting $r \rightarrow \infty$, we obtain the result. 


\subsection{When $\alpha \rightarrow 0$}

We conclude the section by showing that, as $\alpha \rightarrow 0$, the probability to see to infinity from a given point goes to 1 .

Proposition 2. It holds that

$$
\lim _{\alpha \rightarrow 0} \mathbb{P}[\mathfrak{V}=\infty]=1 \text {. }
$$

Proof. We recall first that $\{\mathfrak{V}=\infty\}=\bigcap_{r>0}\left\{Y_{r} \neq \varnothing\right\}$, so that

$$
\mathbb{P}[\mathfrak{V}=\infty]=\lim _{r \rightarrow \infty} \mathbb{P}\left[Y_{r} \neq \varnothing\right]
$$

In view of (13), it is enough to show that, for a fixed $\epsilon \in(0, \pi / 2)$, we have

$$
\lim _{\alpha \rightarrow 0} \liminf _{r \rightarrow \infty} \frac{\mathbb{E}\left[y_{r}(\epsilon)\right]^{2}}{\mathbb{E}\left[y_{r}(\epsilon)^{2}\right]}=1 .
$$

We have

$$
\begin{aligned}
\mathbb{P}\left[\theta, \theta^{\prime} \in Y_{r}\right] & \leq \mathbb{P}\left[L_{r}(\theta) \subset \mathcal{W}\right] \mathbb{P}\left[L_{r}\left(\theta^{\prime}\right) \backslash L_{t_{\left|\theta-\theta^{\prime}\right|}} \subset \mathcal{W}\right] \\
& \leq C(\alpha)^{2} \mathrm{e}^{-\alpha r} \mathrm{e}^{-\alpha\left(0 \vee\left(r-t_{\left|\theta-\theta^{\prime}\right|}\right)\right)} \\
& \leq C(\alpha)^{2} \mathrm{e}^{-2 \alpha r} \mathrm{e}^{\alpha t_{\left|\theta-\theta^{\prime}\right|}}
\end{aligned}
$$

where

$$
C(\alpha)=\exp \left(-\frac{\alpha}{2 \mathbb{E}[\sinh (R)]} \mathbb{E}[2 \pi(\cosh (R)-1)]\right) \rightarrow 1 \quad \text { as } \alpha \rightarrow 0
$$

is the constant that we obtained in the proof of Lemma 1 when calculating $f(r)$. Therefore, for $\epsilon \in(0, \pi / 2)$, we have

$$
\mathbb{E}\left[y_{r}(\epsilon)^{2}\right] \stackrel{(10)}{\leq} C(\alpha)^{2} \mathrm{e}^{-2 \alpha r} \int_{0}^{\epsilon} \int_{0}^{\epsilon} \mathrm{e}^{\alpha t_{\left|\theta-\theta^{\prime}\right|}} \mathrm{d} \theta \mathrm{d} \theta^{\prime} .
$$

Since $\mathbb{E}\left[y_{r}(\epsilon)\right]^{2}=C(\alpha)^{2} \epsilon^{2} \mathrm{e}^{-2 \alpha r}$, it follows that

$$
\frac{\mathbb{E}\left[y_{r}(\epsilon)\right]^{2}}{\mathbb{E}\left[y_{r}(\epsilon)^{2}\right]} \geq \frac{\epsilon^{2}}{\int_{0}^{\epsilon} \int_{0}^{\epsilon} \mathrm{e}^{\alpha t_{\left|\theta-\theta^{\prime}\right|} \mathrm{d} \theta \mathrm{d} \theta^{\prime}} .}
$$

Using (26) and (29), we see that

$$
t_{\left|\theta-\theta^{\prime}\right|} \leq \log \left(\frac{O(1)}{\sqrt{1-\cos \left(2\left|\theta-\theta^{\prime}\right|\right)}}\right) \leq \log \left(\frac{O(1)}{\left|\theta-\theta^{\prime}\right|}\right) .
$$

Hence,

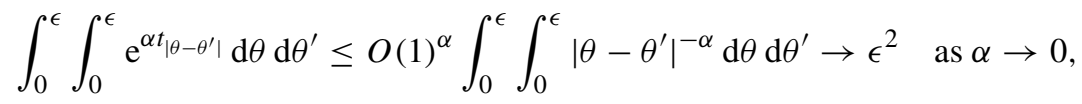

where the limit follows from straightforward calculations. Now (41) follows from (42) and (43) with the choice $\epsilon=\pi / 4$. 


\section{Visibility with varying intensity}

We consider the case where all radii are deterministic, equal to $R>0$. For a fixed intensity, there exists a critical radius $R_{C}=\sinh ^{-1}(1 / 2 \lambda)$ under which visibility to infinity occurs with positive probability. When the radius $R$ goes to 0 , this probability goes to 1 . The question we are interested in this section is the following: what happens when the intensity $\lambda$ of the underlying Poisson point process is a function $\lambda(R)$ of the radius which goes to $\infty$ when $R \rightarrow 0$ ?

Let $\mathfrak{V}_{\lambda, R}$ be the total visibility associated with the choice of $R$ for the radius of the balls and $\lambda$ for the intensity of the underlying Poisson point process. In the following result, we show that we can adapt the intensity so that the maximal visibility will not be higher than a fixed level with high probability.

Theorem 5. For every $r>0$ and $p \in(0,1)$, there exists an explicit functional $\lambda(R)$ given by (48) below such that $\lim _{R \rightarrow 0} \mathbb{P}\left[\mathfrak{V}_{\lambda(R), R} \leq r\right]=p$.

Proof. We denote by $\bar{r}=\tanh (r / 2)$ and $\bar{R}=\tanh (R / 2)$. A ball $B_{\mathbb{H}}(x, R)$ intersects $B_{\mathbb{H}}(0, r)=B_{\mathbb{R}^{2}}(0, \bar{r})$ if and only if $\|x\| \leq \alpha(\bar{r})$, where, for every $\bar{r} \in[0,1]$,

$$
\alpha(\bar{r})=\frac{\sqrt{\left(1-\bar{R}^{2}\right)^{2}+4(\bar{R}+\bar{r})\left(\bar{R}+\bar{R}^{2} \bar{r}\right)}-\left(1-\bar{R}^{2}\right)}{2 \bar{R}(1+\bar{R} \bar{r})} .
$$

The number of such $x \in X$ is Poisson distributed of mean

$$
2 \pi \Lambda=4 \lambda \pi \int_{0}^{\alpha(\bar{r})} \frac{2 \rho}{\left(1-\rho^{2}\right)^{2}} \mathrm{~d} \rho=4 \lambda \pi \frac{\alpha^{2}(\bar{r})}{1-\alpha^{2}(\bar{r})} .
$$

These points are independent and rotation invariant, and the common density of their radial coordinates is

$$
f(\rho)=\frac{4 \lambda}{\Lambda} \mathbf{1}_{[0, \alpha(\bar{r})]}(\rho) \frac{\rho}{\left(1-\rho^{2}\right)^{2}} .
$$

In particular, the (normalized) size $A_{\bar{r}, \bar{R}}$ of the 'shadow' of one such ball $B_{\mathbb{H}}(x, R)$ is equal to

$$
\frac{1}{\pi} \arcsin \left(\bar{R} \frac{1-\|x\|^{2}}{\|x\|(1-\bar{R})^{2}}\right)
$$

if $0 \leq\|x\| \leq \beta(\bar{r})=\sqrt{\left(\bar{R}^{2}+\bar{r}^{2}\right) /\left(\bar{R}^{2} \bar{r}^{2}+1\right)}$ and something smaller if $\beta(\bar{r})<\|x\| \leq \alpha(\bar{r})$. It is easy to check that, when $R \rightarrow 0$, the probability that an $f$-distributed random variable is in $[\beta(\bar{r}), \alpha(\bar{r})]$ goes to 0 . Consequently, we may use (46) combined with (45) to show that $A_{\bar{r}, \bar{R}} / \bar{R}$ converges in distribution to a limit distribution.

The probability of the event $\left\{\mathfrak{V}_{\lambda, R} \leq r\right\}$ is equal to the probability to cover the Euclidean circle centered at the origin and of radius $\bar{r}$ by a Poisson number of mean $2 \pi \Lambda$ of i.i.d. random arcs such that their normalized lengths are distributed as $A_{\bar{r}, \bar{R}}$. We are going to use a slightly modified version of an original result due to Janson [9, Lemma 8.1]: for every $\Lambda, \varepsilon>0$, let $p_{\Lambda, \varepsilon}$ be the probability of covering the circle of perimeter one with a Poisson number of mean $2 \pi \Lambda$ of independent and uniformly located random arcs with a half length distributed as $\varepsilon \widetilde{\mathcal{R}}_{\Lambda}$, $\widetilde{\mathcal{R}}_{\Lambda}$ being a bounded random variable for every $\Lambda$. If

1. $\widetilde{\mathcal{R}}_{\Lambda} \rightarrow \widetilde{\mathcal{R}}$ in distribution as $\Lambda \rightarrow \infty$, where $\widetilde{\mathcal{R}}$ is a random variable with a finite moment of order $1+\varepsilon$ for some $\varepsilon>0$, and 
2. $\varepsilon$ (going to 0 ) and $\Lambda$ (going to $\infty$ ) are related such that the convergence

$$
\lim _{\epsilon \rightarrow 0, \Lambda \rightarrow \infty}\{2 \pi b \varepsilon \Lambda+\log (b \varepsilon)-\log (-\log (b \varepsilon))\}=t
$$

holds, where $b:=\mathbb{E}[\widetilde{\mathcal{R}}] / \pi$,

then the probability $p_{\Lambda, \varepsilon}$ goes to $\exp \left(-\mathrm{e}^{-t}\right)$.

We apply the above result with the choice $\varepsilon=\bar{R}, \Lambda$ given by (44), and $t$ such that $\exp \left(-\mathrm{e}^{-t}\right)=p$. In this case, the length $\widetilde{\mathcal{R}}_{\Lambda}$ is equal to $A_{\bar{r}} \bar{R} / \bar{R}$. Because of what has been explained above, $\widetilde{\mathcal{R}}_{\Lambda}$ converges to the limit in distribution of (46), i.e. the limit $\widetilde{\mathcal{R}}$ is distributed as $\left(1-U^{2}\right) / \pi U$, where $U$ is $f$-distributed. In particular, $\mathbb{E}\left[\widetilde{R}^{1+\varepsilon}\right]<\infty$ for every $0 \leq \varepsilon<1$. With the choice

$$
\lambda(R)=\frac{1-\alpha^{2}(\bar{r})}{2 \alpha^{2}(\bar{r})}\left[-\frac{\log (\bar{R})}{2 \pi b \bar{R}}+\frac{\log (-\log (\bar{R}))}{2 \pi b \bar{R}}+\frac{t-\log (b)}{2 \pi b \bar{R}}\right],
$$

we verify that (47) is satisfied and we deduce from the covering result due to Janson that

$$
\lim _{R \rightarrow 0} \mathbb{P}\left[\mathfrak{V}_{\lambda(R), R} \leq r\right]=p
$$

\section{Acknowledgements}

Special thanks are due to the referees for their careful reading which has greatly improved the paper.

\section{References}

[1] Ballani, F. (2006). On second-order characteristics of germ-grain models with convex grains. Mathematika 53, 255-285.

[2] Benjamini, I., Jonasson, J., Schramm, O. and Tykesson, J. (2009). Visibility to infinity in the hyperbolic plane, despite obstacles. ALEA Latin Amer. J. Prob. Math. Statist. 6, 323-342.

[3] Calka, P. (2002). The distributions of the smallest disks containing the Poisson-Voronoi typical cell and the Crofton cell in the plane. Adv. Appl. Prob. 34, 702-717.

[4] Calka, P., Michel, J. and Porret-Blanc, S. (2011). Asymptotics of the visibility function in the Boolean model. Preprint. Available http://arxiv.org/abs/0905.4874v2.

[5] Cannon, J. W., Floyd, W. J., Kenyon, R. and Parry, W. R. (1997). Hyperbolic geometry. In Flavors of Geometry (Math. Sci. Res. Inst. Publ. 31), Cambridge University Press, pp. 59-115.

[6] Hall, P. (1988). Introduction to the Theory of Coverage Processes. John Wiley, New York.

[7] Heinrich, L. (1998). Contact and chord length distribution of a stationary Voronoŭ tessellation. Adv. Appl. Prob. 30, 603-618.

[8] Herman, I., Melançon, G. and Marshall, M. S. (2000). Graph visualization and navigation in information visualization: a survey. IEEE Trans. Visual. Comput. Graphics 6, 24-43.

[9] JANSON, S. (1986). Random coverings in several dimensions. Acta Math. 156, 83-118.

[10] Jonasson, J. (2008). Dynamical circle covering with homogeneous Poisson updating. Statist. Prob. Lett. 78, 2400-2403.

[11] Kahane, J.-P. (1985). Some Random Series of Functions (Camb. Stud. Adv. Math. 5), 2nd edn. Cambridge University Press.

[12] Kahane, J.-P. (1990). Recouvrements aléatoires et théorie du potentiel. Colloq. Math. 60/61, 387-411.

[13] Kahane, J.-P. (1991). Produits de poids aléatoires indépendants et applications. In Fractal Geometry and Analysis (Montreal, PQ, 1989; NATO Adv. Sci. Inst. Ser. C Math. Phys. Sci. 346), Kluwer, Dordrecht, pp. 277-324.

[14] Last, G. and Schassberger, R. (2001). On the second derivative of the spherical contact distribution function of smooth grain models. Prob. Theory Relat. Fields 121, 49-72.

[15] Lovász, L. (2009). Very large graphs. In Current Developments in Mathematics, Int. Press, Somerville, MA, pp. 67-128. 
[16] LyOns, R. (1996). Diffusions and random shadows in negatively curved manifolds. J. Funct. Anal. 138, $426-448$.

[17] Meester, R. ANd Roy, R. (1996). Continuum Percolation (Cambr. Tracts Math. 119). Cambridge University Press.

[18] Porret-Blanc, S. (2007). Sur le caractère borné de la cellule de Crofton des mosaïques de géodésiques dans le plan hyperbolique. C. R. Math. Acad. Sci. Paris 344, 477-481.

[19] Santaló, L. A. (1976). Integral Geometry and Geometric Probability (Encyclopedia Math. Appl. 1). AddisonWesley, Reading, MA.

[20] Tykesson, J. (2007). The number of unbounded components in the Poisson Boolean model of continuum percolation in hyperbolic space. Electron. J. Prob. 12, 1379-1401.

[21] Ungar, A. A. (2008). Analytic Hyperbolic Geometry and Albert Einstein's Special Theory of Relativity. World Scientific, Hackensack, NJ. 